\title{
Journal of Psychiatry and Behavioral Sciences
}

Open Access | Review Article

\section{Effect of omega-3 fatty acids on offending behavior in repeat violent offenders: $A$ randomized controlled trial feasibility study}

\author{
Laura Miles $^{1 *}$; Melanie Simpson ${ }^{2}$; Tony Butler ${ }^{2}$; Lisa G Wood ${ }^{3}$; Lee Knight ${ }^{2}$; David Greenberg ${ }^{4}$; Peter Schofield ${ }^{1}$ \\ ${ }^{1}$ Hunter New England Mental Health Neuropsychiatry Service, Australia \\ ${ }^{2}$ Kirby Institute, UNSW Sydney, Australia \\ ${ }^{3}$ School of Biomedical Sciences and Pharmacy, University of Newcastle, Australia \\ ${ }^{4}$ Justice Health \& Forensic Mental Health Network, Australia
}

\section{*Corresponding Author(s): Laura Miles,} Neuropsychiatry Service, Hunter New England Local Health District, Newcastle, NSW PO BOX 833 Newcastle NSW 2300 Australia

Tel: +6124033 5701,

Email: Laura.Miles@hnehealth.nsw.gov.au

Received: Mar 26, 2017

Accepted: Apr 20, 2018

Published Online: Apr 27, 2018

Journal: Journal of Psychiatry and Behavioral Sciences

Publisher: MedDocs Publishers LLC

Online edition: http://meddocsonline.org/

Copyright: (C) Miles L (2018). This Article is distributed under the terms of Creative Commons Attribution 4.0 international License

Keywords: Omega-3 polyunsaturated fatty acid; Randomised Controlled Trial; Violence; Community based offenders; Retention

\section{Abstract}

Evidence suggests that certain behavioral problems, including a tendency towards impulsive-violence, might arise in part as a consequence of a potentially correctable, dietary imbalance of essential fatty acids in susceptible individuals. This study aimed to determine the feasibility of conducting a Randomized Controlled Trial (RCT) using omega-3 polyunsaturated fatty acid supplementation in a community sample of impulsive, repeat-violent offenders. Behavioral measures of impulsivity, anger, depression, irritability and self-reported offending were examined. Participants were randomized to a 12 week placebocontrolled trial of Omega-3 polyunsaturated fatty acid supplementation. Eligible participants were recruited from the Newcastle Courthouse, New South Wales (Australia), reported a minimum of two prior violent offences, and scored highly on the 15-item Barratt Impulsiveness Scale. Forty-four individuals commenced the trial, with 22 completing the 12 week intervention. There was a statistically significant mean increase in Omega-3 Index among participants who received the intervention. At week 12, participants who self-reported offending in the past month had a significantly lower mean Omega-3 Index compared to those who reported no offending behavior. This study demonstrates that community-based individuals with histories of violent offending who self-reported reoffending within the past month had a lower mean Omega-3 Index compared to similar individuals who reported no offending behavior. This finding suggests diets of repeat violent offenders lacking important essential fatty acids may contribute to behavioral problems. Violent offender recruitment, adherence and techniques for improving lost to follow up are discussed. An adequately powered RCT of this intervention is warranted.

Cite this article: Miles L, Simpson M, Butler T, Wood LG, Knight L, et al. Effect of omega-3 fatty Acids on offending behaviour in repeat violent offenders: A randomized controlled trial feasibility study. J Psychiatry Behav Sci. 2018; 1: 1002. 


\section{Introduction}

Violence is a leading cause of death and disability and profoundly impacts victims and the community. For every person killed by violence, an estimated 20-40 individuals receive injuries requiring hospitalization [1]. In Australia, assaults comprise over $82 \%$ of all violent crimes, while intimate partner violence is estimated to contribute to $7.9 \%$ of the total burden of disease among women $18-44$ years old [2,3]. Programs and interventions to reduce the incidence of violence and its consequences are needed given the high level of violence in the community.

A compelling body of evidence has emerged which suggests that certain psychiatric and behavioral states, including a tendency toward impulsive-violence, might arise in part from a minor, potentially correctable, dietary imbalance in relation to so-called 'essential fatty acids' in susceptible individuals [4]. Omega-3 and omega-6 Polyunsaturated Fatty Acids (PUFAs) comprise two families of fatty acids which are required for normal growth and development [5]. These fatty acids are incorporated into phospholipids that comprise cellular membranes throughout the body and mediate a range of vital physiological effects [6-8].

A number of studies, some conducted in custodial settings, have shown that omega-3 PUFA supplementation can lead to improved self-regulation and reductions in aggression, anger and anti-social behavior [9-12]. Two Randomized Controlled Trials (RCTs) have demonstrated a significant reduction (26\% and $34 \%$ respectively) in anti-social behavior among incarcerated adolescents receiving a supplement compared to those receiving a placebo $[10,12,13]$ have recently demonstrated a significant negative correlation between behavioral measures of aggressive behavior (and manifestations of Attention Deficit Hyperactivity Disorder) and the omega-3 index (the sum of Eicosapentaenoic Acid (EPA) and Docosahexaenoic Acid (DHA) expressed as percent of total erythrocyte fatty acids) among a cohort of prison inmates.

However, no study to date has investigated the potential benefits of omega-3 PUFA supplementation in an adult offender population in the community where the benefits would have a larger, more 'public' impact over those conducted in closed settings such as prison. We conducted a double-blind randomized placebo-controlled trial over three months to determine the feasibility of conducting an intervention with impulsive community-based offenders with histories of violence and to examine the impact of omega-3 PUFA on recidivism and behavioral changes (impulsivity, anger, depression, irritability).

\section{Methods}

This double-blind randomised placebo-controlled trial was conducted between February and December 2015 in Newcastle, New South Wales (NSW), Australia. Eligible participants were randomly assigned by an independent statistician to receive either the placebo ( $98 \%$ paraffin, $2 \%$ fish Oil (flavour)/capsule) or the Omega-3 PUFA supplement (400 mg EPA, $210 \mathrm{mg}$ DHA/capsule). Participants were directed to take five capsules per day for 12 weeks. All participants were monitored over the phone at weeks one, three and nine, and attended face-to-face interviews at baseline, week six and week 12. Participants were supplied with a six weeks supply of capsules at baseline and week six.

Participants were approached at the Newcastle Courthouse on the date of their court appearance. Access to offenders was made available following discussions with New South Wales Department of Justice and the Newcastle Local Court. Interested offenders provided recruitment personnel their preferred telephone number and were contacted within 5 days following their court appearance. Participants were pre-screened for eligibility and if the criteria were met offered an appointment at the Hunter New England Mental Health building located on the Newcastle Calvary Mater Hospital Campus. Mobile phone numbers were provided by 150 (97\%) of the interested offenders, highlighting the accessibility for immediate contact. To minimize loss to follow-up, multiple contact numbers and/or email addresses were obtained.

Eligible participants were men 18 years old and over who had a minimum of two convictions for violent offences, and who scored a minimum of 35 on the 15 -item version of the Barratt Impulsiveness Scale (BIS-15) during the pre-screening telephone call. Participants had to provide informed consent, be fluent in English and be willing to provide a sample of blood at the beginning and end of the study.

Participants were excluded if they did not meet the above criteria, had an allergy to seafood or iodine, were currently using anti-coagulants, anti-inflammatories, or antidepressant medication, had a severe mental illness (schizophrenia, bipolar disorder, major depression), were considered to be at high risk of suicide, if they had significant renal or hepatic impairment, were anticipating a custodial sentence, or moving interstate or to a remote area preventing follow-up. Individuals who had a conviction for murder or for child sex offences were excluded.

Violent crime was defined as per the Australian and New Zealand Standard Offence Classification (ANZSOC) and included the following five categories: homicide (excluding murder but including manslaughter); acts intended to cause injury; dangerous and negligent acts endangering persons; abduction, harassment and other offences against the person; and robbery, extortion and related offences.

All participants provided informed consent and received $\$ 40$ cash per face-to-face interview for reimbursement of time and parking. The study received ethical approval through the Hunter New England Human Research Ethics Committee (14/08/20/4.06). The trial was registered with the Australian and New Zealand Clinical Trials Registry (ACTRN12616000220460).

\section{Instruments and assessments}

Behavioral measures of impulsivity (15-item version of the Barratt Impulsiveness Scale and Barratt Impulsivity Scale-30 item [14] and International Personality Disorder Examination [15], anger (State Trait Anger Expression Inventory) [16] and the Anger Irritability Assault Questionnaire (AIAQ) [17], depression (Beck Depression Inventory-II [18], irritability (AIAQ), psychological distress (Kessler Psychological Distress Scale (K-10) [19]), wellbeing (the Short Form-12 Health Survey (SF-12) [20] were administered at three time points over three months (baseline, 6 and 12 weeks).

The baseline and week 12 face-to-face interviews took approximately 1.5 hours to administer, while the week six interviews in which half the instruments were administered took 30 minutes (BIS-30, STAXI, AIAQ, BECK, K-10 and SF-12). At baseline questions about the participants' medical history including head injuries and treatment for drug or alcohol problems, literacy measures (Wechsler Individual Achievement Test) [1], prison history and other demographic data were also 
collected.

Participants were contacted by phone at weeks one, three and nine to monitor compliance and to inquire if any side effects had been experienced. When participants reported forgetting to take the supplements the research coordinator offered to send an automated text reminder and suggested tips for remembering to take the supplement. Prior to the six and 12 week face-to-face interviews, the research coordinator sent a courtesy text message reminder of the appointment to participants' mobile phones. Many participants reported progress and schedule changes through text messaging. Adherence to the daily regimen of five capsules was monitored by the research coordinator counting the remaining capsules at the six weekly visits and by participant self-report.

\section{Data Analysis}

Independent t-tests were used to compare means between participants who completed the study to those who did not. $T$ tests were used to examine mean differences in instrument scores between those in the omega-3 PUFA and placebo groups. Chi squared tested were used to examine differences in proportions between the treatment and placebo groups and those for whom we lost contact. Loss to follow up prevented analysis within groups compared to baseline. Dried blood spot testing blood analysis was performed by Omega Quant, LLC USA, on whole blood drops collected at baseline and week 12. All analyses were conducted using SPSS Version 23.

\section{Results}

A total of 155 men attending the Newcastle Courthouse were assessed between February and September 2015 for study eligibility; 111 were excluded from the study. Of those, 73 people did not meet the eligibility criteria and 38 declined to participate. The most common reasons for not meeting the eligibility criteria were having less than two violent offences $(n=20)$, current use of antidepressant medication or a low score $(<35)$ on the BIS-15 Form ( $n=22)$. Forty-four participants were randomized into the study. Thirty participants completed both the baseline and week six interviews, and 22 (50\%) completed the 12 week intervention. Overall, 22 out of 82 eligible individuals $(27 \%)$ completed the intervention. The reasons participants gave for failing to complete the study included: job commitments $(n=2)$, transportation issues $(n=1)$, and returning to custody $(n=3)$ however the majority of non-completers did not explain why they withdrew from the study and were lost to follow up $(n=16)$ (Figure 1).

Participants' median age was 31 years (range: $18-53$ years); over three-quarters of the sample (77\%) were under 39 years old. The majority of participants (61\%) reported at least one head injury with loss of consciousness over their lifetime, with many reporting multiple head injuries. The average literacy score was 120 , which is equivalent to a year 10 education or that of a 16 year old.

Among the 22 who completed the trial, there was a statistically significant increase from baseline to week 12 in mean Omega-3 Index ( $p=0.002$ ) among those receiving the omega-3 PUFA supplementation, consistent with good adherence to the medication (Table 1). As expected, there was no increase in Omega-3 Index from baseline to 12 weeks among those in the placebo group. An association was apparent between selfreported adherence and the Omega-3 Index (Figure 2). The Omega-3 Index of two participants randomized to the Omega-3
PUFA arm did not increase over time.

At baseline, participants reported committing property crime ( $n=16,37 \%)$, dealing drugs $(n=16,37 \%)$, fraud $(n=2,4 \%)$, and violence $(n=14,33 \%)$ in the past month. Results on the Beck Depression Inventory were consistent with mild mood disturbances in $22.7 \%(n=10)$, borderline clinical depression in $15.9 \%$ $(n=7)$, moderate depression in $6.8 \%(n=3)$, severe depression in $22.7 \%(n=10)$ and extreme depression in one participant (2.3\%). At baseline, those participants randomized to the Omega-3 PUFA supplementation group had significantly higher levels of nervousness $(p=0.011)$, irritability $(p=0.038)$, and verbal assault scores $(p=0.044)$ compared to the placebo group. The treatment group was also significantly more likely to be alcohol dependent $(p=0.031)$, and reported having taken more drugs than they had planned in the past 12 months ( $p=0.045$ ). No differences existed between the Omega-3 PUFA or Placebo groups in regards to baseline impulsivity scores $(p=0.683)$ or criminal offending $(p=0.252)$. The baseline characteristics by treatment group among participants who completed the study are shown (Table 4).

The Omega-3 PUFA group had a significantly higher mean psychological distress score compared to participants taking the placebo $(p=0.030)$ at week 12 . They reported more feelings of hopelessness $(p=0.028)$, being restless $(p=0.011)$ or fidgety $(p=0.007)$ and worthless $(p=0.015)$ compared to the placebo group. Although there was no significant difference between the groups in self-reported criminal activity at week 12 $(p=0.074)$ (Table 2), blood analysed at week 12 demonstrated a significantly higher Omega-3 Index among participants who did not commit crime, compared to those who did commit crime $(p=0.037)$ (Table 3). The mean Omega-3 Index of those who had not committed crime at week 12 was $6.17 \%(\mathrm{~N}=14)$ compared to $4.36 \%(\mathrm{~N}=8)$ among those who did reported offending. Analysis of the mean impulsivity score among the participants who completed the study showed no significant change between scores from baseline compared to week 12 ( $p=0.477)$.

When comparing participants who completed the study with those who did not, mean baseline impulsivity scores, literacy scores, alcohol and drug dependency were not found to be statistically different. Among all participants, $82 \%$ were drug dependent. In the past 12 months, the most commonly used drugs were: ICE (methamphetamine) (39\%) and cannabis (36.6\%). In the same period, $51 \%$ reported ICE as being the drug which had caused the biggest problems. Examination of the demographic variables demonstrated no statistical differences between age, ethnic background, education level, income source, marital status, head trauma, or smoking status among participants who completed the trial compared to those who did not. Those who did not complete the study were more likely to have been convicted of a crime and sent to prison $(p=0.014)$.

\section{Discussion}

No study to date has investigated the feasibility of Omega-3 PUFA supplementation in impulsive-violent community offenders with histories of violent offending. This study demonstrated that at least one quarter of repeat violent offenders living in the community who were approached and who met the eligibility criteria for the study were willing to participate in a randomized trial and adhere to an investigational regimen for up to three months. Furthermore, the Omega-3 Index in those who received Omega-3 PUFA supplementation provided evidence that adherence was maintained by the majority of participants who 
completed the trial.

Public health advances in the community have followed from the identification and treatment of dietary deficiencies. Thyroid disease, neural tube defects, tooth decay and a range of neurologic, and cardiac disorders essentially disappeared once the cause was recognized and appropriate supplementation programs were developed for those at risk [21-23]. There are significant gaps in our knowledge regarding the relationship between diets and offending behavior, especially for those individuals in the community. In our study, using Omega-3 PUFA supplementation to reduce behavioral problems has shown some promising results, particularly in regards to its possible impact on self-reported re-offending.

In our study, at baseline there were no differences in selfreported criminal offending between participants who completed the trial and those who did not. However, blood analysis of all participants at week 12 demonstrated those who reported no criminal activity in the past month had a significantly higher Omega-3 Index than those who did report criminal activity over the preceding month. This observation is in agreement with several RCTs, the first of which found that dietary supplementation including vitamins, minerals, omega- 6 and omega-3 PUFAs reduced antisocial behavior in incarcerated young male offenders [10]. Another study conducted among Dutch young adult offenders, showed that aggressive and rule-breaking behaviors were reduced in the experimental group receiving vitamins, minerals, omega-3 and omega-6 PUFA compared to the control group over a 3 month period [12]. Another study conducted in a community-based sample of 24 substance abusers found that those with a history of assaultive behavior had a dietary history of less fish and lower daily PUFA intake [9]. The sample size in the current study is small, and this finding needs to be interpreted with caution, however our data suggests the need for larger studies.

The results suggest that it would be both feasible and valuable to conduct an RCT with a much larger sample of community dwelling offenders. Such a study would enable better matching of potentially significant confounding factors such as baseline anger levels and drug/alcohol dependencies across study intervention arms.

This study was designed to assess the feasibility of conducting a study within this difficult population. Below we outline in detail the challenges and successes we experienced, in the hope that this information may interest and assist other investigators.

A major challenge faced by the research team was the recruitment of impulsive repeat-violent offenders throughout the 12 week study period. A series of ineffective methods were first used to recruit participants. These included advertising online through Gumtree, Facebook and in the local newspaper. Study personnel also met with local barristers, mailed recruitment information to solicitors and support services for disadvantaged people, none of which resulted in a participant. Direct recruitment at the Newcastle Courthouse was most effective on days when large numbers of offenders were processed by the court.

The sex, age and personality of recruiting personnel, as well as court jurisdiction and court type could be contributing factors in successful voluntary recruitment. Understanding when most offenders would be seen at the court was essential for maximizing recruitment in the current study.
While recruiting at the Newcastle Courthouse many potential participants reported a fear of needles and initially refused to participate until research staff emphasized that blood was to be collected by finger prick. Dried blood spot testing was selected as opposed to drawing venous blood due to the cost of employing a phlebotomist or training research staff in phlebotomy, the costs of storage space and handling of samples, and the risk of losing interested participants due to phobias. Previous studies have found a high correlation ( $r=0.97$ ) between dried blood spot and red blood cell methods for determining the Omega-3 Index [24].

In our study, flexibility (of appointment times, days, places) by the study team was crucial for building rapport and maintaining contact. Given the demonstrated wide use of mobile phones among offenders, a review of SMS messaging services and applications for use in this population could also benefit future studies in reducing loss to follow-up.

Most participants in our study demonstrated an interest in contributing to health research, particularly during the first interview. As the study progressed into the 2 nd and 3rd interviews, our experience has shown that participants develop a fatigue to repetitive questions, particularly during lengthy visits. The research survey questions are important to understand change over time but become redundant to the participant. To reduce question fatigue we suggest less than 1 hour visits and employing various interactive methods for capturing the information (phones, other electronic devices, face to face interviewing, and supported pencil to paper responses).

High drop-out rates of $50 \%$ or more are not uncommon in populations such as offenders, such that substantial oversampling is necessary to achieve the required power, increasing resource usage significantly [25].

Another recommendation, would be to include a trial period of observation for eligible participants prior to randomizing them into study treatment groups. For interventions, such as conventional pharmacotherapy, where there are no lasting effects of the drug once ceased (unlike the current study which the effects of omega 3 PUFA might persist for some time), an actual run-in period is a possible approach, whereby all participants are initially given the active intervention prior to randomization (after which typically $50 \%$ would enter the placebo arm). The majority of participants who left the current study did so by week six. Randomization after a short trial period would potentially have the effect of identifying participants who are more likely to adhere to a regimen for the length of the study.

Ongoing drug and alcohol dependence in community based violent offenders creates challenges for recruitment and retention, and can raise obstacles when submitting studies for ethical approval.

The barriers to attending appointments such as unreliable transport (many offenders legally lose driver's licenses), rigid work commitments, loss of contact (many offenders change phones regularly to avoid being tracked/lose phones or have no phone credit), and missed appointments (many offenders experience memory issues) may be offset through providing monetary reimbursements or a variety of gift cards/vouchers $[26,27]$.

The various options for compensating offenders may be challenged by human ethics committees and the research is often critically reviewed as "incentive driven": committees may re- 
strict reimbursements or require that payment be in the form of vouchers or gift cards so as not to support drug or alcohol addictions. These restrictions are not imposed on other study populations and as such could be regarded as discriminatory.

Across study populations, and particularly within Australia, there is a need to investigate the various forms of reimbursement which will compensate participants adequately for their time, knowledge/experiences and costs associated (e.g. parking, travel) to attend the study. An understanding of which form of reimbursement, the appropriate cash value, the frequency in which it is given over time and the association with participant response rates would provide a guideline for future studies.

Populations such as repeat violent offenders come with a multitude of complex substance dependencies, behavioral issues and criminal histories. Researchers would be well placed to have interview training for collecting sensitive information (crimes not officially reported) without putting themselves or the participant in an ethical predicament in terms of legal requirements to report undetected crimes to the police.

Setting up pathways for researchers to access professional support where needed following offender contact would be advisable. Direct measures for incorporating design features which promote the personal safety of staff may include selfdefense training and exercising the use of personal and on-site alarms. While self-defense training empowers research staff to react appropriately or deter immediate physical threats, regular contact with staff is the best form of support. Importantly, it should be noted that no researcher was physically threatened during the conduct of this study.

In the present study, it seemed as though issues that the participants raised with the researcher (LM) were not being addressed by health workers to whom the participants potentially had access. Many participants requested individual feedback or advice for handling anger, parenting, drug addictions and anxiety following survey questions. A mechanism for providing staggered feedback at each follow up appointment based on survey responses in the form of a graph or summary information may provide insight into behaviors or capabilities and encourage participants to return for follow up.

Similarly, future studies examining offender health need to incorporate methods to guide researchers to know when and how to initiate a referral for services is advisable. In our study, most participants reported a dependence on drugs and/or alcohol, with most believing their use/abuse was not problematic.

A systematic response for offering public and private drug and alcohol treatment options to participants may provide an opportunity to address these significant health issues. Similarly, a system for referring participants to psychologists or councilors would be advised as the offenders often became emotional when reflecting upon traumatic experiences during the survey administration. In both instances providing the participant with either type of support (feedback or referral) is an ethically responsible practice, however the study design considerations should take into account the impact of the type support on future participation responses.

The results of this feasibility study indicated that community-based repeat-violent offenders would participate in an intervention study of omega-3 over a period of up to three months. A significant loss to follow up should be expected, when screening for impulsive men with complex substance dependencies and behavioral issues.

Twenty-eight percent of eligible repeat violent offenders from the local Courthouse volunteered to participate in a 12 week randomized trial. Half of the participants who joined, completed the trial even with impulsive behaviors, substance dependencies, mood disturbances and criminal histories. Treatment adherence was objectively demonstrated by a mean increase in the Omega 3 Index among those participants on the active preparation $(p=0.002)$. Finally, there was a statistically significant difference in past month self-reported criminal activity among those who adhered with the Omega-3 PUFA supplementation and had a corresponding increase in Omega-3 Index.

We outlined recommendations and provided practical solutions to improve participant retention while highlighting overarching ethical considerations with offender populations. An adequately powered RCT among community based offenders would minimize confounding factors seen across the intervention arms within our small sample. We hope the challenges and successes we experienced, may interest and assist other investigators in the design of future offender health research.

\section{Acknowledgements}

The Aboriginal Cardiovascular Omega-3 Trial at the South Australian Health and Medical Research Institute for donating the Omega-3 and placebo, NSW Court Services, Department of Justice, and Newcastle Courthouse for access to potential participants.

\section{Figures}

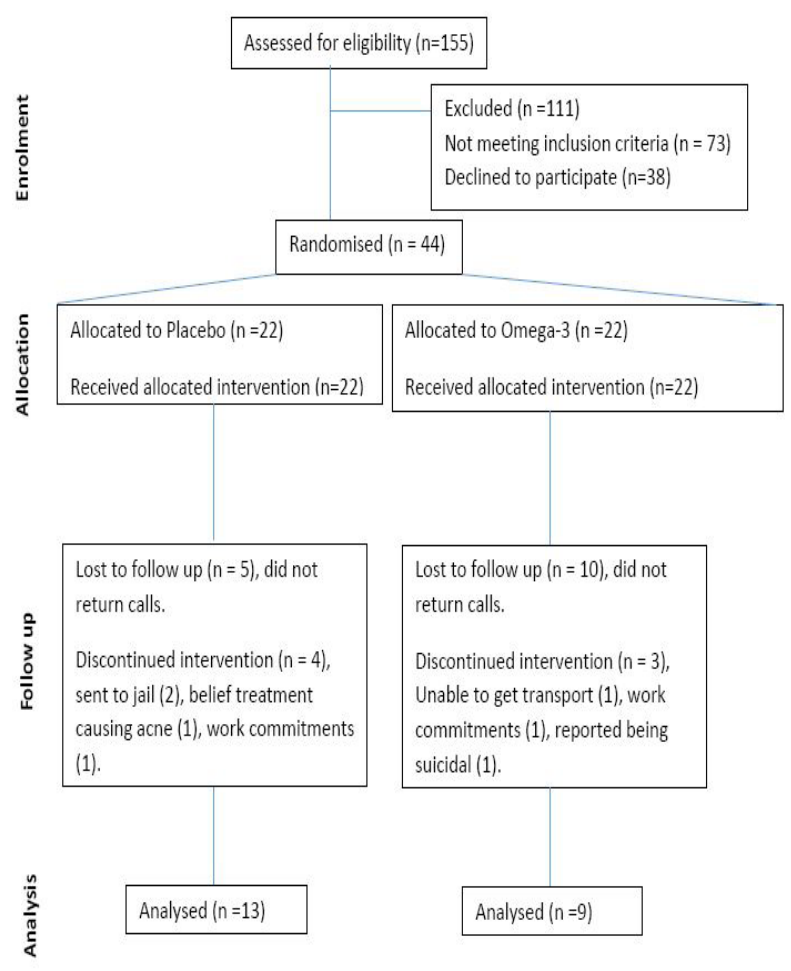

Figure 1: Flow Diagram of the Progress through the Phases of Enrolment, Treatment Allocation to Analysis. 


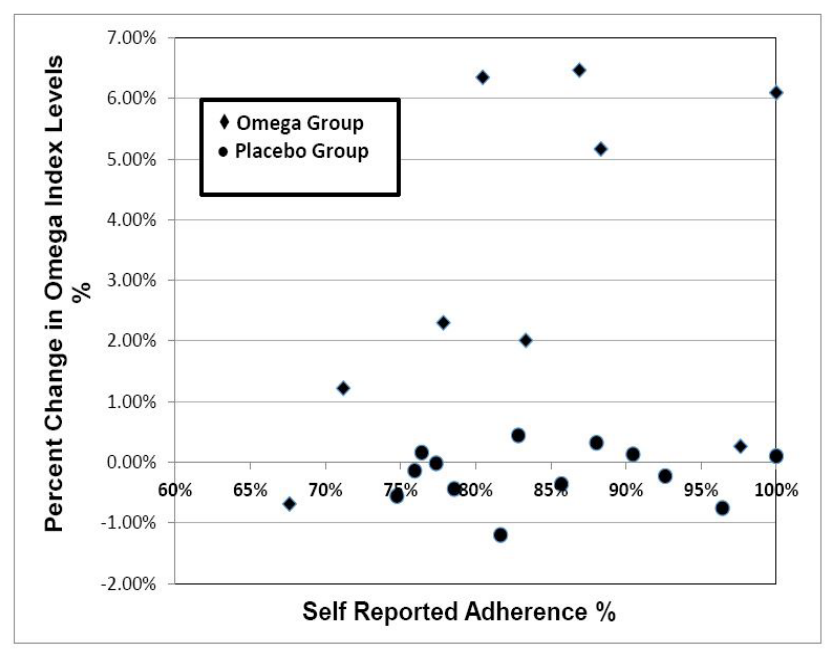

Figure 2: Percent Change in Omega-3 Index Plotted against SelfReported Adherence to Daily Regimen

Note: Omega-3 Index is a measure of omega-3 fatty acids, EPA +DHA in red blood cells and is reported as a cumulative percentage. Percent change is the calculated difference between baseline and week 12 Omega-3 Index. Adherence is self-reported by participant.

\section{Tables}

Table 1: Omega-3 Index Levels at Baseline and Week 12 by Treatment Group

\begin{tabular}{|c|c|c|c|c|c|c|c|c|c|}
\hline & \multicolumn{6}{|c|}{ Treatment Group } & \multirow{3}{*}{$\begin{array}{c}95 \% \mathrm{Cl} \text { for } \\
\text { Mean } \\
\text { Difference }\end{array}$} & \multirow{3}{*}{$\mathrm{t}$} & \multirow{3}{*}{$\mathrm{df}$} \\
\hline & \multicolumn{3}{|c|}{ Placebo } & \multicolumn{3}{|c|}{ Omega-3 Supplement } & & & \\
\hline & M & SD & $\mathrm{n}$ & M & SD & $\mathrm{n}$ & & & \\
\hline Baseline & 4.04 & 0.60 & 21 & 4.22 & 0.65 & 23 & $-0.20,0.56$ & 0.94 & 42 \\
\hline Week 12 & 4.26 & 0.44 & 13 & 7.33 & 3.03 & 9 & $1.30,4.83$ & $3.63 *$ & 20 \\
\hline
\end{tabular}

Note: Omega- 3 Index is a measure of omega- 3 fatty acids, EPA+DHA, in red blood cells. ${ }^{*} p=.002$, statistically significant $p<.05$

Table 2: Self-Reported Criminal Activity at Week 12 by Treatment Group

\begin{tabular}{|c|c|c|c|c|}
\hline \multirow{2}{*}{} & \multicolumn{4}{|c|}{ Self-Reported Crime by Treatment Group } \\
\cline { 2 - 5 } & \multicolumn{3}{|c|}{$\begin{array}{c}\text { Placebo } \\
\mathrm{n}=13\end{array}$} & $\begin{array}{c}\text { Omega-3 Supplement } \\
\mathrm{n}=9\end{array}$ \\
\cline { 2 - 5 } & Value & $\mathrm{df}$ & $\begin{array}{c}\text { Asymtomatic Sig } \\
(2-\text { sided })\end{array}$ & $\begin{array}{c}\text { Exact Sig } \\
\text { (2-sided) }\end{array}$ \\
\hline Pearson Chi-Square & $4.19^{\mathrm{a}}$ & 1 & 0.4 & \\
\hline Continuity Correction & 2.55 & 1 & 0.11 & 0.074 \\
\hline Fisher's Exact Test & & & & \multicolumn{3}{|c|}{} \\
\hline
\end{tabular}

Note: 2 cells have expected cell count less than $5 . p=.074$, not statistically significant $p<.05$

Table 3: Omega-3 Index by Self-Reported Criminal Activity

\begin{tabular}{|c|c|c|c|c|c|c|c|c|c|}
\hline & \multicolumn{6}{|c|}{ Self-Reported Crime } & \multirow{3}{*}{$\begin{array}{l}95 \% \mathrm{Cl} \text { for } \\
\text { Mean } \\
\text { Difference }\end{array}$} & \multirow{3}{*}{$\mathrm{t}$} & \multirow{3}{*}{$d f$} \\
\hline & \multicolumn{3}{|c|}{ No } & \multicolumn{3}{|c|}{ Yes } & & & \\
\hline & M & SD & $\mathrm{n}$ & M & SD & $\mathrm{n}$ & & & \\
\hline a'Baseline & 4.39 & 0.73 & 16 & 4.01 & 0.53 & 27 & $-0.76,0.01$ & -1.94 & 41 \\
\hline b'Week 12 & 6.17 & 2.87 & 14 & 4.36 & 0.51 & 8 & $-3.50,-0.12$ & $-2.30 *$ & 14 \\
\hline
\end{tabular}

'Baseline: Includes all participants' baseline Omega Index levels by self-reported crime in past 6 months. $p=.059$, statistically significant $p<.05$

${ }^{b}$ Week 12: Participants Omega-3 Index at week 12 by self-reported crime in past 4 weeks, equal variances not assumed. ${ }^{*} p=.037$, statistically significant $p<.05$ 
Table 4: Baseline Characteristics by Treatment Group among completers

\begin{tabular}{|c|c|c|c|c|}
\hline & \multicolumn{2}{|c|}{$\begin{array}{l}\text { Placebo } \\
n=13\end{array}$} & \multicolumn{2}{|c|}{$\begin{array}{l}\text { Omega-3 Supplement } \\
\qquad \mathrm{n}=9\end{array}$} \\
\hline & Frequency & Percent & Frequency & Percent \\
\hline \multicolumn{5}{|c|}{ Age Group } \\
\hline $18-26$ & 5 & 38.5 & 2 & 22.2 \\
\hline $27-36$ & 4 & 30.8 & 4 & 44.4 \\
\hline $37+$ & 4 & 30.8 & 3 & 33.3 \\
\hline \multicolumn{5}{|c|}{ Highest Level of Education } \\
\hline Under year 9 & 2 & 15.4 & 2 & 22.2 \\
\hline $9-10$ & 9 & 69.2 & 6 & 66.7 \\
\hline $11-12$ & 2 & 15.4 & 1 & 11.1 \\
\hline \multicolumn{5}{|c|}{ Main Income } \\
\hline Criminal & 2 & 15.4 & 1 & 11.1 \\
\hline Employment & 3 & 23.1 & 3 & 33.3 \\
\hline Government Pension & 3 & 23.1 & 4 & 44.4 \\
\hline Other & 5 & 38.5 & 1 & 11.1 \\
\hline \multicolumn{5}{|c|}{ aSent to Prison } \\
\hline Yes & 4 & 30.8 & 4 & 44.4 \\
\hline No & 9 & 69.2 & 5 & 55.6 \\
\hline \multicolumn{5}{|c|}{ Sustained Head Injury } \\
\hline Yes & 6 & 46.2 & 6 & 66.7 \\
\hline No & 7 & 53.8 & 3 & 33.3 \\
\hline
\end{tabular}

a Participants who were sent to prison previously were significantly more unlikely to complete the study period.

\section{References}

1. Wechsler D. Wechsler Individual Achievement Test - Second Edition (WIAT-II). Retrieved from San Antonio: World Health Organization. World Report on Violence and Health. 2002.

2. Australian Bureau of Statistics. Recorded Crime - Offenders, 2015-16. Canberra, Australia. 2017.

3. Vos T, Astbury J, Piers LS, Magnus A, Heenan M, Stanley L, Webster K. Measuring the impact of intimate partner violence on the health of women in Victoria, Australia. Bulletin of the World Health Organization. 2006; 84: 739-744.

4. Ross B, Seguin J, Sieswerda LE. Omega-3 fatty acids as treatments for mental illness: which disorder and which fatty acid? Lipids Health Dis. 2007; 6: 1186-1121.

5. Heinrich SC. Dietary Omega-3 fatty acid supplementation for optimizing neuonal structure and function. Molecular Nutrition and Food Research. 2010; 54: 477-486.

6. Kliewer SA, Sundseth SS, Jones SA, Brown PJ, Wisely GB, Koble $\mathrm{CS}$, Lehmann JM. Fatty acids and eicosanoids regulate gene expression through direct interactions with peroxisome proliferator-activated receptors $\alpha$ and $\gamma$. Proceedings of the National Academy of Sciences of the United States of America. 1997; 94: 4318-4323.

7. Salam N, Litman B, Kim HY, Gawrisch K. Mechanisms of action of docosahexaenoic acid in the nervous system. Lipids. 2001; 36: 945-959.

8. Tassoni D, Kaur G, Weisinger RS, Sinclair AJ. The role of eicosanoids in the brain. Asia Pacific Journal of Clinical Nutrition. 2008; 17: 220-228.

9. Buydens-Branchey L, Branchey M. Long-chain n-3 polyunsaturated fatty acids decrease feelings of anger in substance abusers. Psychiatry Research. 2008; 15: 95-104.
10. Gesch B, Hammond SM, Hampson SE, Eves A, Crowder MJ. Influence of supplementary vitamins, minerals and essential fatty acids on the antisocial behaviour of young adult prisoners. Randomised, placebo-controlled trial. British Journal of Psychiatry. 2002; 181: 22-28.

11. Hamazaki T, Sawazaki S, Itomura M, Asaoka E, Nagao Y, Nishimura $\mathrm{N}$, Kobayashi $\mathrm{M}$. The effect of docosahexaenoic acid on aggression in young adults. A placebo-controlled double-blind study. The Journal of Clinical Investigation. 1996; 15: 1129-1133.

12. Zaalberg A, Nijman H, Bulten E, Stroosma L, van der Staak C. Effects of nutritional supplements on aggression, rule-breaking, and psychopathology among young adult prisoners. Aggressive Behavior. 2010; 36: 117-126.

13. Meyer BJ, Byrne MK, Collier C, Parletta N, Crawford D, Winberg PC, Grant L. Baseline Omega-3 Index Correlates withAggressive and Attention Deficit DisorderBehaviours in Adult Prisoners. PLoS One. 2015; 10.

14. Barratt E, Stanford MS. Impulsiveness. In C. G. Costello (Editor), Personality Characteristics of the Personality Disordered Client. New York: Wiley. 1995.

15. Loranger AW, Janca A, Sartorius N. Assessment and diagnosis of personality disorders: The ICD-10 International Personality Disorder Examination (IPDE). Cambridge, U.K: Cambridge University Press. 1997.

16. Spielberger CD. STAXI-2: State-Trait Anger Expression Inventory-2: professional manual. Odessa, FL (P.O. Box 998, Odessa 33556: Psychological Assessment Resources. 1999.

17. Coccaro EF, Astill JL, Herbert J, Schut A. Fluoxetine treatment of impulsive aggression in DSM-III-R personality disorder patients. Journal of Clinical Psychopharmacology. 1990; 10: 373-375.

18. Beck AT, Steer RA, Brown GA. Beck Depression Inventory-II. San 
Antonio: The Psycholgical Corporation. 1996.

19. Kessler RC, Barker PR, Colpe LJ, Epstein JF, Gfroerer JC, Hiripi E, Zaslavsky AM. Screening for serious mental illness in the general population. Archives of General Psychiatry. 2003; 60: 184-189.

20. Ware JE, Kosinski MA, Keller SD. Sf-12: How to Score the sf-12 Physical and Mental Health Summary Scales. Lincoln: Quality Metric Incorporated. 1998.

21. Dean HT, Arnold FA, Jay P, Kknutson JW. Studies on mass control of dental caries through fluoridation of the public water supply. Public Health Reports. 1950; 65: 1403-1408.

22. Hetzel BS. lodine deficiency disorders (IDD) and their eradication. Lancet. 1983; 2: 1126-1129.

23. Smithells RW, Nevin NC, Seller MJ, Sheppard S, Harris R, Read AP, Wild J. Further experience of vitamin supplementation for the prevention of neural tube defect recurrences. Lancet. 1983; 321: 1027-1031.

24. Johnston DT, Deuster PA, Harris WS, MacRae H, Dretsch MN. Red blood cell omega-3 fatty acid levels and neurocognitive performance in deployed U.S. Servicemembers. Nutritional Neuroscience. 2013; 16: 30-38.
25. Brorson HH, Anrnevik EA, Rand-Hendriksen K, Duckert F. Drop out from addiction treatment: A systematic review of risk factors. Clinical Psychology Review. 2013; 33: 1010-1024.

26. Festinger DS, Marlowe DB, Dugosh KL, Croft JR, Arabia PL. Higher Magnitude Cash Payments Improve Research Follow-up Rates Without Increasing Drug Use or Perceived Coercion. Drug and Alcohol Dependence. 2008; 96: 128-135.

27. Thurstone C, Salomensen-Sautel S, Riggs PD. How adolescents with substance use disorder spend research payments. Drug and Alcohol Dependence. 2010; 111: 262-264. 